\title{
Decay of Correlations under Dobrushin's Uniqueness Condition and its Applications
}

\author{
H. Künsch \\ Mathematics Department, ETH Zentrum, CH-8092 Zürich, Switzerland
}

\begin{abstract}
An estimate on the correlation of functionals of Gibbs fields satisfying Dobrushin's uniqueness condition is given. As a consequence a result of Gross saying that the truncated pair correlation function decays in the same weighted summability sense as the potential can be extended to the whole Dobrushin uniqueness region. Applications to the central limit theorem and the second derivative of the pressure are also given.
\end{abstract}

\section{Introduction}

The well-known uniqueness theorem of Dobrushin [3] states that there is only one Gibbs state if the interaction is weak which means that the temperature is high or the activity small. This theorem has the advantage of being very general. No condition like finite range, pair interactions, finiteness of the single spin space or translation invariance is needed. Despite its generality the condition is surprisingly sharp as shown by Simon [12]. Moreover one gets not only uniqueness from it, but also properties of the Gibbs state: Dobrushin [4] showed that it is uniformly mixing, and Gross [6], [7] proved results on the decay of correlation and on the differentiability of the pressure. However one of his results, Theorem 2 in [6], was not proved in the whole Dobrushin uniqueness region, and his expression for the second derivative of the pressure in [7] is different from the ususal covariance series. In our paper here we close these two gaps.

In Sect. 2 we recall results from Dobrushin [4] in the form we will need them later. In Sect. 3 we state then our main result on the decay of correlation (Theorem 3.2). As corollaries we get the results of Gross [6] in the whole Dobrushin uniqueness region. In Sect. 4 we apply our results to check known conditions for the central limit theorem for functionals of Gibbs fields, and in Sect. 5 we show that the second derivative of the pressure is equal to the usual covariance series. The main theorems are proved in Sect. 6 by an extension of Dobrushin's uniqueness proof in [4]. We do not construct a dynamics which has the Gibbs state as an invariant measure like in Vasershtein [13] and Gross [6]. Finally in Sect. 7 we 
formulate our results for a general Vasershtein distance which covers also interesting non-compact examples.

\section{Notations and Definitions}

Let $L$ be a countable set of sites, $X$ a compact metric space, the individual spin space, and $\Omega=X^{L}$ the configuration space containing all functions $s: L \rightarrow X$. For $M \subset L$ we denote by $\mathscr{F}_{M}$ the $\sigma$-field generated by the maps $s \rightarrow s_{a}, a \in M$. Instead of $\mathscr{F}_{L}$ we write simply $\mathscr{F}$. The set of all continuous real-valued functions on $\Omega$ (with respect to the product topology) is denoted by $C(\Omega)$. For $f \in C(\Omega)$ and $a \in L$ put

$$
\rho_{a}(f)=\sup \{|f(s)-f(t)|, s=t \text { except at } a\} .
$$

For such $f$ the following holds for all $s, t \in \Omega$ :

$$
|f(t)-f(s)| \leqq \sum_{a \in L} \rho_{a}(f)
$$

Let $\mathscr{V}$ be the class of finite non-empty subsets $V$ of $L$ and let $\left(p^{V}\right)_{V \in \mathscr{V}}$ be a specification on $\Omega$. By this we mean that for each $V p^{V}(A \mid s)\left(A \in \mathscr{F}_{V}, s \in X^{L \mid V}\right)$ is a probability kernel, i.e. a probability in the first and a measurable function in the second argument. For many purposes it is convenient to introduce the associated kernels $\pi^{V}(A \mid s)(A \in \mathscr{F}, s \in \Omega)$ which are uniquely defined by the following properties (compare Preston [10], Chapter 1):

$\pi^{V}(A \mid \cdot)$ is $\mathscr{F}_{L \backslash V}$-measurable,

$\pi^{V}(\cdot \mid s)$ is a probability on $(\Omega, \mathscr{F})$ which coincides with

the Diract measure $\delta_{s}$ on $\mathscr{F}_{L \backslash V}$ and with $p^{V}\left(\cdot \mid s_{L \backslash V}\right)$ on $\mathscr{F}_{V}$.

We will always assume that the specification is continuous in the sense that $\pi^{V} f \in C(\Omega)$ for all $f \in C(\Omega)$, where

$$
\pi^{V} f(s)=\int \pi^{V}(d t \mid s) f(t)
$$

A Gibbs state to a specification $\left(p^{V}\right)_{V \in \mathcal{V}}$ is a probability $\mu$ on $(\Omega, \mathscr{F})$ whose conditional distribution with respect to $\mathscr{F}_{L \backslash V}$ are given by $\pi^{V}$, i.e.

$$
\mu\left[A \mid \mathscr{F}_{L \backslash V}\right](\cdot)=\pi^{V}(\mathrm{~A} \mid \cdot) \mu \text {-a.s. } \quad(A \in \mathscr{F}, V \in \mathscr{V}),
$$

or equivalently

$$
\mu\left(\pi^{V} f\right)=\mu(f)(f \in C(\Omega), V \in \mathscr{V}) .
$$

The set of all Gibbs states is denoted by $\mathscr{G}(p) \cdot \mathscr{G}(p)$ is convex and compact in the weak topology if $\left(p^{V}\right)_{V \in \mathcal{V}}$ is continuous. In order to prove the existence of Gibbs states we must assume that the specification is consistent which means that

$$
\pi^{V}\left(\pi^{W} f\right)=\pi^{V} f \quad(f \in C(\Omega), W \subset V \in \mathscr{V}) .
$$

By compactness arguments the weak limit of $\left(\pi^{V_{n}}(\cdot \mid s)\right)_{n \in \mathbb{N}}$ exists for $s \in \Omega$ and $V_{n} \uparrow L$ 
(at least if we choose a suitable subsequence), and using (1.7) it is easy to see that the limit is in $\mathscr{G}(p)$.

By a potential we mean a family of continuous $\mathscr{F}_{V}$-measurable functions $\varphi_{V}: \Omega \rightarrow \mathbb{R}, V \in \mathscr{V}$, satisfying

$$
\sum_{V \ni a}|V| \sup \left\{\left|\varphi_{V}(s)\right|, s \in \Omega\right\}<\infty \quad(a \in L)
$$

Let $v$ be a finite measure on $X$, the a priori single-spin measure. The case of main interest is when all $p^{V}(d t \mid s)$ are absolutely continuous with respect to the product measure $\prod_{a \in V} v\left(d t_{a}\right)$ and the density is given by a potential:

$$
p^{V}(d t \mid s)=Z_{V}(s)^{-1} \exp \left(-\sum_{W \cap V \neq \phi} \varphi_{W}(t s)\right) \prod_{a \in V} v\left(d t_{a}\right) .
$$

Here $Z_{V}(s)$ is a normalizing constant and $t s$ is the configuration which is equal to $t$ in $V$ and equal to $s$ in $L \backslash V$. Such specifications are always continuous and consistent. However the potentials will be used only in Sect. 5.

If $X$ is not compact, then all the results which follow can be proved in the same way: we only have to take instead of $C(\Omega)$ an appropriate class of functions. However the hypotheses we will make are never satisfied in the interesting examples with a non-compact $X$. In order to deal with such examples it is useful to introduce a general Vasershtein distance as it was done in Dobrushin [4]. In Sect. 7 we will briefly state our results in this more general situation.

\section{Comparison of Gibbs States and Uniqueness}

Let $\left(p_{i}^{V}\right)_{V \in \mathscr{V}}, i=1,2$, be two continuous specifications and put

$$
\begin{aligned}
& \gamma_{a b}=\frac{1}{2} \sup \left\{\left\|p_{i}^{b}(\cdot \mid s)-p_{i}^{b}(\cdot \mid t)\right\|_{\mathrm{Var}}, s=t \text { except at } a, i=1,2\right\}(a \neq b \in L), \\
& \gamma_{a a}=0(a \in L), \\
& \beta_{a}=\frac{1}{2} \sup \left\{\left\|p_{1}^{a}(\cdot \mid s)-p_{2}^{a}(\cdot \mid s)\right\|_{\mathrm{Var}}, s \in X^{L \backslash a}\right\} \quad(a \in L) .
\end{aligned}
$$

Let $\Gamma$ be the infinite matrix $\left(\gamma_{a b}\right)_{a, b \in L}$ and $\Gamma_{V}$ its restriction to $a \in V, b \in V$. We put

$$
\begin{array}{ll}
\chi_{a b}^{V}=\sum_{n=0}^{\infty}\left(\Gamma_{V}^{n}\right)_{a b} \quad(a, b \in V \in \mathscr{V}), \\
\chi_{a b}=\sum_{n=0}^{\infty}\left(\Gamma^{n}\right)_{a b} \quad(a, b \in L) .
\end{array}
$$

$\chi_{a b}\left(\chi_{a b}^{V}\right)$ is nothing else than the Green's function (of the set $V$ ) for the random walk on $L$ with transition probabilities $\gamma_{a b}$ provided $\sum_{a} \gamma_{a b} \leqq 1$.

The next theorem is essentially contained in Theorem 3 of Dobrushin [4]:

Theorem 2.1. Suppose $\left(p_{i}^{V}\right)_{V \in \mathscr{V}}, i=1,2$, are two continuous specifications such that 
$\sum_{a} \gamma_{a b} \leqq \alpha<1$ and $\mu_{i} \in \mathscr{G}\left(p_{i}\right), i=1,2$. Then for all $f \in C(\Omega)$ we have

$$
\left|\mu_{1}(f)-\mu_{2}(f)\right| \leqq \sum_{a, b} \beta_{a} \chi_{a b} \rho_{b}(f)
$$

Remark 2.2 It will become clear in the proof that $\sum_{a} \gamma_{a b} \leqq \alpha<1$ can be replaced by the slightly weaker condition $\sum_{a} \chi_{a b}<\infty(b \in L)$. However we will state all our results with the former condition which is easier to check.

The proof will be given in Sect. 6. We will state now some consequences of Theorem 2.1. Whenever we have only one specification $\left(p^{V}\right)_{V \in \mathscr{V}}$ we will take an analogous definition of $\gamma_{a b}$ as in (2.1) just omitting all indices $i$.

Corollary 2.3 (Dobrushin). Let $\left(p^{V}\right)_{V \in \mathcal{V}}$ be a continuous specification with $\sum_{a} \gamma_{a b} \leqq$ $\alpha<1$. Then $|\mathscr{G}(p)| \leqq 1$.

Proof. Let $\mu_{1}$ and $\mu_{2}$ be in $\mathscr{G}(p)$. Then $\beta_{a}=0$ for all $a \in L$, whence $\mu_{1}(f)=\mu_{2}(f)$ for all $f \in C(\Omega)$, i.e. $\mu_{1}=\mu_{2}$.

Corollary 2.4. Let $\left(p^{V}\right)_{V \in \mathcal{V}}$ be a continuous and consistent specification with $\sum_{a} \gamma_{a b} \leqq$ $\alpha<1$. Then for any sequence $V_{n}^{\dagger} L$ and any $s \in \Omega \pi^{V n}(\cdot \mid s)$ converges weakly to the unique Gibbs state $\mu$, and the difference $\mu(f)-\pi^{V} f(s)$ can be estimated by formula (2.4) for $f$ in $C(\Omega)$ with $\sum_{b} \rho_{b}(f)<\infty$.

Proof. Fix $V \in \mathscr{V}$ and $s \in \Omega$. By the consistency condition $(1.7) p^{V}(\cdot \mid s)$ is a Gibbs state on $\Omega_{V}=X^{V}$ to the specification $\left(p^{W}(\cdot \mid \cdot s)\right)_{W \subset V}$. Therefore by Theorem 2.1 for $f \in C(\Omega)$

$$
\begin{aligned}
\left|\pi^{V} f(t)-\pi^{V} f(s)\right| & \left.\leqq\left|\int f(u t)\left(p^{V}(d u \mid t)-p^{V}(d u \mid s)\right)\right|+\mid \int f(u t)-f(u s)\right) p^{V}(d u \mid s) \mid \\
& \leqq \sum_{a \in V, b \in V}\left(\sum_{c \notin V} \gamma_{c a}\right) \chi_{a b}^{V} \rho_{b}(f)+\sum_{c \notin V} \rho_{c}(f) .
\end{aligned}
$$

Therefore also

$$
\left|\mu(f)-\pi^{V} f(s)\right| \leqq \sum_{a, b \in V}\left(\sum_{c \notin V} \gamma_{c a}\right) \chi_{a b}^{V} \rho_{b}(f)+\sum_{c \notin V} \rho_{c}(f) .
$$

Now for all $a \in L \sum_{c \notin V} \gamma_{c a}$ tends to zero for $V \uparrow L$, and

$$
\sum_{a, b \in V}\left(\sum_{c \notin V} \gamma_{c a}\right) \chi_{a b}^{V} \rho_{b}(f) \leqq \alpha \sum_{a, b \in V} \chi_{a b}^{V} \rho_{b}(f) \leqq \alpha(1-\alpha)^{-1} \sum_{b} \rho_{b}(f) .
$$

Therefore by dominated convergence $\pi^{V} f(s) \rightarrow \mu(f)$ for $f$ in $C(\Omega)$ with $\sum_{b} \rho_{b}(f)<\infty$. Such $f$ generate weak convergence.

From the estimate (2.3) we can easily deduce an estimate of the uniform mixing rate of the Gibbs state $\mu$ : 
Proposition 2.5. (Dobrushin). Let $\mu$ be the unique Gibbs state to a consistent and continuous specification $\left(p^{V}\right)_{V \in \mathcal{V}}$ with $\sum_{a} \gamma_{a b} \leqq \alpha<1$. Then for all $W \subset V \in \mathscr{V}$ we have

$$
\varphi(W, V)=\sup \left\{|\mu(A \mid B)-\mu(A)|, A \in \mathscr{F}_{W}, B \in \mathscr{F}_{L \backslash V}, \mu(B) \neq 0\right\} \leqq \sum_{b \in W}\left(\sum_{c \notin V, a \in V} \gamma_{c a} \chi_{a b}\right)
$$

which tends to zero for $W$ fixed and $V \uparrow L$.

Proof. It is sufficient to prove the inequality for open sets $A \in \mathscr{F}_{W}$. Approximating $f=1_{A}$ by a suitable sequence of continuous functions we find from (2.3)

$$
\left|p^{V}(A \mid s)-p^{V}(A \mid t)\right| \leqq \sum_{a \in V, b \in W}\left(\sum_{c \notin V} \gamma_{c a}\right) \chi_{a b}^{V}
$$

So for $B \in \mathscr{F}_{L \backslash V}$ we get

$$
\begin{aligned}
|\mu(A \cap B)-\mu(A) \mu(B)| & =\left|\int 1_{B}(s) p^{V}(A \mid s) \mu(d s)-\iint 1_{B}(s) p^{V}(A \mid t) \mu(d t) \mu(d s)\right| \\
& \leqq \mu(B) \sum_{a \in V, b \in W}\left(\sum_{c \notin V} \gamma_{c a}\right) \chi_{a b}^{V} .
\end{aligned}
$$

Because $\chi_{a b}^{V} \leqq \chi_{a b}$ the inequality is proved. The convergence to zero follows from dominated convergence: For fixed $a \in L \sum_{c \notin V} \gamma_{c a}$ goes to zero for $V \uparrow L$, and for fixed $b \in W$

$$
\sum_{a \in V} \sum_{c \notin V} \gamma_{c a} \chi_{a b} \leqq \alpha \sum_{a} \chi_{a b} \leqq \alpha(1-\alpha)^{-1}
$$

\section{Decay of Correlation}

It is well known that the uniform mixing coefficients $\varphi(W, V)$ which were estimated in Proposition 2.5 tell us something about the correlation of functions localized in disjoint domains. Namely if $f$ and $g$ are in $C(\Omega)$ and $f$ is $\mathscr{F}_{W}$-measurable and $g \mathscr{F}_{L \backslash V}$-measurable, then we have

$$
\left|\operatorname{Cov}(f, g)_{\mu}\right| \leqq 2 \varphi(W, V)\|f\|_{\infty}\|g\|_{\infty} .
$$

In order to put this into a more intuitive appealing form it is convenient to consider a semimetric $d$ on $L$, i.e. $d(\cdot, \cdot)$ is a nonnegative symmetric function on $L \times L$ for which the triangle inequality holds.

Proposition 3.1. Let $\mu$ be the unique Gibbs state to a consistent and continuous specification $\left(p^{V}\right)_{V \in \mathcal{V}}$ satisfying $\sum_{a} \gamma_{a b} e^{d(a, b)} \leqq \alpha<1$ for some semimetric $d$. Then for $f$ and $g$ in $C(\Omega), f \mathscr{F}_{W}$-measurable and $g \mathscr{F}_{V}$-measurable with $d(W, V)=\inf \{d(a, b)$, $a \in W, b \in V\}>0$

$$
\left|\operatorname{Cov}(f, g)_{\mu}\right| \leqq e^{-d(W, V)}|W| 2 \alpha(1-\alpha)^{-1}\|f\|_{\infty}\|g\|_{\infty} .
$$

Proof. Let $\widetilde{V}$ be the set $\{a \in L, d(W, a)<d(W, V)\}$. Then using (3.1) and Proposition 2.5 with $\tilde{V}$ instead of $V$ we have

$$
\left|\operatorname{Cov}(f, g)_{\mu}\right| \leqq 2 \sum_{b \in W}\left(\sum_{c \notin \tilde{V}, a \in \tilde{V}} \gamma_{c a} \chi_{a b}\right)\|f\|_{\infty}\|g\|_{\infty} .
$$


Now for $b \in W, a \in \tilde{V}, c \notin \tilde{V}: e^{d(W, V)} \leqq e^{d(b, c)} \leqq e^{d(b, a)} e^{d(a, c)}$. Therefore

$$
\begin{aligned}
\left|\operatorname{Cov}(f, g)_{\mu}\right| & \leqq 2 e^{-d(W, V)} \sum_{b \in W}\left(\sum_{c \notin \tilde{V}, a \in \tilde{V}} \gamma_{c a} e^{d(c, a)} \chi_{a b} e^{d(a, b)}\right)\|f\|_{\infty}\|g\|_{\infty} \\
& \leqq 2 e^{-d(W, V)}|W| \alpha \sup \left\{\sum_{a} \chi_{a b} e^{d(a, b)}, b \in L\right\}\|f\|_{\infty}\|g\|_{\infty} .
\end{aligned}
$$

But $\sum_{a} \gamma_{a b} e^{d(a, b)} \leqq \alpha<1$ implies by the triangle inequality

$$
\sum_{a} \chi_{a b} e^{d(a, b)} \leqq(1-\alpha)^{-1}
$$

The next theorem which is our main result improves Proposition 3.1 in two ways: First it applies also to functions not necessarily localized in disjoint domains, and second we will get a decay of correlation at the same speed as the coefficients $\gamma_{a b}$. Let us define

$$
\gamma^{*}=\sup \left\{\left(2 \chi_{a a}-1\right)^{-1}, a \in L\right\} \leqq 1
$$

Theorem 3.2. Let $\mu$ be the unique Gibbs state to a continuous and consistent specification $\left(p^{V}\right)_{V \in \mathcal{V}}$ with $\sum_{a} \gamma_{a b} \leqq \alpha<1$. Then for any two functions $f$ and $g$ in $C(\Omega)$

$$
\left|\operatorname{Cov}(f, g)_{\mu}\right| \leqq \gamma^{*} \sum_{a, b}\left(\sum_{c} \chi_{c a} \chi_{c b}\right) \rho_{a}(f) \rho_{b}(g) .
$$

We will give the proof together with the proof of Theorem 2.1 in Sect. 6. Let us give now two corollaries of it which extend results of Gross [6] (see also the Remark 3.5i) below).

Corollary 3.3. Let $\mu$ be the unique Gibbs state to a continuous and consistent specification $\left(p^{V}\right)_{V \in \mathcal{V}}$ satisfying $\sum_{a} \gamma_{a b} e^{d(a, b)} \leqq \alpha<1$ for some semimetric $d$ on L. Then for any two functions $f$ and $g$ in $C(\Omega)$ and any points $a, b \in L$

$$
\left|\operatorname{Cov}(f, g)_{\mu}\right| \leqq e^{-d(a, b)} \gamma^{*}(1-\alpha)^{-2} \sum_{c} e^{d(a, c)} \rho_{c}(f) \sum_{c} e^{d(b, c)} \rho_{c}(g) .
$$

Proof. By the triangle inequality we find

$$
\begin{aligned}
e^{d(a, b)} \sum_{a^{\prime}, b^{\prime}}\left(\sum_{c} \chi_{c a^{\prime}} \chi_{c b^{\prime}}\right) \rho_{a^{\prime}}(f) \rho_{b^{\prime}}(g) \leqq & \sum_{a^{\prime}, b^{\prime}}\left(\sum_{c} \chi_{c a^{\prime}} e^{d\left(c, a^{\prime}\right)} \chi_{c b^{\prime}} e^{d\left(c, b^{\prime}\right)}\right) \\
& \cdot e^{d\left(a, a^{\prime}\right)} \rho_{a^{\prime}}(f) e^{d\left(b, b^{\prime}\right)} \rho_{b^{\prime}}(g) .
\end{aligned}
$$

Furthermore by (3.2) $\sum_{c} \chi_{c a^{\prime}} e^{d\left(c, a^{\prime}\right)} \chi_{c b^{\prime}} e^{d\left(c, b^{\prime}\right)} \leqq \sum_{c} \chi_{c a^{\prime}} e^{d\left(c, a^{\prime}\right)} \cdot \sum_{c} \chi_{c b^{\prime}} e^{d\left(c, b^{\prime}\right)} \leqq(1-\alpha)^{-2}$. So the corollary follows immediately from Theorem 3.2.

Corollary 3.4. Assume $L=\mathbb{Z}^{v}$. Let $\mu$ be the unique Gibbs state to a continuous and consistent specification $\left(p^{V}\right)_{V \in \mathscr{V}}$ satisfying $\sum_{a} \gamma_{a b} e^{d(a, b)} \leqq \alpha<1$ and $\sum_{b} \gamma_{a b} e^{d(a, b)} \leqq \gamma<1$ 
for some translation invariant semimetric $d$ on $\mathbb{Z}^{\nu}$. Then for any functions $f$ and $g$ in $C(\Omega)$ and any point $b \in \mathbb{Z}^{v}$

$$
\sum_{a}\left|\operatorname{Cov}\left(f, g \circ \theta_{a}\right)_{\mu}\right| e^{d(b, a)} \leqq \gamma^{*}(1-\alpha)^{-1}(1-\gamma)^{-1} \sum_{a} e^{d(b, a)} \rho_{a}(f) \cdot \sum_{a} e^{d(0, a)} \rho_{a}(g) .
$$

Here $\theta_{a}: \Omega \rightarrow \Omega$ is the shift operator defined by $\left(\theta_{a} s\right)_{b}=s_{a+b}$.

Proof. Using Theorem 3.2 and formula (3.4) we get

$$
\begin{aligned}
\sum_{a}\left|\operatorname{Cov}\left(f, g \circ \theta_{a}\right)_{\mu}\right| e^{d(b, a)} \gamma^{*-1} \leqq & \sum_{a, a^{\prime}, b^{\prime}}\left(\sum_{c} \chi_{c a^{\prime}} e^{d\left(c, a^{\prime}\right)} \chi_{c b^{\prime}} e^{d\left(c, b^{\prime}\right)}\right) \\
& \cdot e^{d\left(b, a^{\prime}\right)} \rho_{a^{\prime}}(f) e^{d\left(a, b^{\prime}\right)} \rho_{b^{\prime}}\left(g \circ \theta_{a}\right) .
\end{aligned}
$$

But since $d\left(a, b^{\prime}\right)=d\left(0, b^{\prime}-a\right)$ and $\rho_{b^{\prime}}\left(g \circ \theta_{a}\right)=\rho_{b^{\prime}-a}(g)$, we can first take the sum over $a$ and then the corollary follows immediately using (3.2).

Remarks 3.5. i) In the Corollaries 3.3 and 3.4 we have smaller constants than Gross [6] in his Theorems 1 and 2, and - what is more important-Corollary 3.4 holds under a weaker condition: We request only $\alpha<1$ and $\gamma<1$ while Gross needs $\alpha<1$ and $\gamma(1+\alpha)<1$.

ii) In Corollary 3.4 the case $d \equiv 0$ is also of great interest. In the applications in Sect. 4 and 5 we will use only this case.

iii) If $L=\mathbb{Z}^{v}$ and $\left(p^{V}\right)_{V \in \mathcal{V}}$ is of finite range, i.e. $p^{a}(A \mid s)$ depends only on $s_{a+b}, b \in N$, for some $N \in \mathscr{V}$, then $\sum_{a} \gamma_{a b} \leqq \alpha<1$ implies $\sum_{a} \gamma_{a b} \exp (\varepsilon|a-b|) \leqq \alpha^{\prime}<1$ if $\varepsilon$ is small enough.

(iv) Assume $L=\mathbb{Z}^{v}$ and the specification $\left(p^{V}\right)_{V \in \mathcal{V}}$ is translation invariant. Then $\gamma_{a b}=\gamma_{a-b}$, so in particular $\alpha=\gamma$. Assume moreover that $\sum_{a} \gamma_{a}<1$ and $\sum_{a} \gamma_{a}|a|^{\beta}<\infty$ for some $\beta>0$. We then consider the metric $d_{\varepsilon, \beta}(a, b)=\min (\varepsilon|a-b|, \beta \log (1+$ $|a-b|))$. It is not hard to see that for $\varepsilon$ small enough $\sum_{a} \gamma_{a} \exp \left(d_{\varepsilon, \beta}(a, 0)\right)<1$. Therefore we have in Proposition 3.1 at least asymptotically a decay like dist $(W, V)^{-\beta}=\inf \{|a-b|, a \in W, b \in V\}^{-\beta}$, and from Corollary 3.4 it follows that $\sum_{a}\left|\operatorname{Cov}\left(f, g \circ \theta_{a}\right)_{\mu}\right||a|^{\beta}<\infty$ for $f$ and $g$ in $C(\Omega)$ with $\sum_{a} \rho_{a}(f)|a|^{\beta}<\infty$ and $\sum_{a} \rho_{a}(g)|a|^{\beta}<\infty$.

\section{The Central Limit Theorem}

In this section we always assume that $L=\mathbb{Z}^{v}$ and the specification $\left(p^{V}\right)_{V \in \mathcal{V}}$ is translation invariant. Then $\gamma_{a b}=\gamma_{a-b}$ and $\alpha=\gamma=\sum_{a} \gamma_{a}$. Moreover if there is only on Gibbs state, all its finite dimensional distributions are also translation invariant.

Let us denote the cube $\left\{a=\left(a_{1}, \ldots, a_{v}\right) \in \mathbb{Z}^{v},-n \leqq a_{i} \leqq n, i=1, \ldots, v\right\}$ by $V_{n}$. We investigate here when the central limit theorem holds, i.e. for which functions $f$ 
and probabilities $\mu$

$$
S_{n}^{*}(f)=(2 n)^{-v / 2} \sum_{a \in V_{n}}\left(f \circ \theta_{a}-\mu(f)\right)
$$

converge weakly to a normal law. This is known to be true if $f$ is $\mathscr{F}_{V}$-measurable for some $V \in \mathscr{V}$ and $\mu$ is a Gibbs state to a specification with weak interaction, see Dobrushin-Tirozzi [5], Sect. 1.3. We give here precise conditions on the specification without assuming finite range, and we allow also for general $f \in C(\Omega)$.

Theorem 4.1. Let $\mu$ be the unique Gibbs state to a translation invariant, continuous and consistent specification $\left(p^{V}\right)_{V \in \mathscr{V}}$ with $\sum_{a} \gamma_{a}<1$ and let $f$ be in $C(\Omega)$. If $\sum_{a} \gamma_{a}|a|^{v+\delta}<\infty$ for $a \delta>0$ and $\sum_{a} \rho_{a}(f)|a|^{v}<\infty$, then $S_{n}^{*}(f)$ converges weakly to a Gaussian random variable with mean zero and variance $\sum_{a} \operatorname{Cov}\left(f, f \circ \theta_{a}\right)_{\mu}$.

Proof. If $f$ is $\mathscr{F}_{V}$-measurable for some $V \in \mathscr{V}$, then Proposition 3.1 and Remark $3.5 \mathrm{iv}$ ) imply that the conditions of Bolthausen [2] for the central limit theorem are satisfied. Equivalently we can also use Proposition 2.5 and adapt Nahapetian's result [8] to the case of bounded variables. For a general $f \in C(\Omega)$ we fix $s \in \Omega$ and approximate $f$ by $f^{m}$, where $f^{m}(t)=f\left(t_{V_{m}} s_{\mathbb{Z}^{v} V_{m}}\right)$. Because $f$ is continuous, $\operatorname{Cov}\left(f^{m}, f^{m} \circ \theta_{a}\right)_{\mu}$ converges to $\operatorname{Cov}\left(f, f \circ \theta_{a}\right)_{\mu}$ for $m \rightarrow \infty$ and fixed $a \in \mathbb{Z}^{v}$, and since $\rho_{a}\left(f^{m}\right) \leqq \rho_{a}(f)$, we have by Theorem 3.2 that $\left|\operatorname{Cov}\left(f^{m}, f^{m} \circ \theta_{a}\right)\right| \leqq \sum_{c, a^{\prime}, b} \chi_{c-a^{\prime}} \chi_{c-b}$ $\rho_{a^{\prime}}(f) \rho_{b-a}(f)$. Therefore by dominated convergence the normal law $\mathscr{N}(0$, $\left.\sum_{a} \operatorname{Cov}\left(f^{m}, f^{m} \circ \theta_{a}\right)_{\mu}\right)$ converges weakly to the normal law $\mathcal{N}\left(0, \sum_{a} \operatorname{Cov}\left(f, f \circ \theta_{a}\right)_{\mu}\right)$.

Furthermore by Corollary $3.4 \lim _{n} \operatorname{Var}\left(S_{n}^{*}(f)-S_{n}^{*}\left(f^{m}\right)\right)_{\mu}=\mid \sum_{a} \operatorname{Cov}\left(\left(f-f^{m}\right)\right.$, $\left.\left(f-f^{m}\right) \circ \theta_{a}\right)_{\mu} \mid \leqq \gamma^{*}(1-\alpha)^{-2}\left(\sum_{a} \rho_{a}\left(f-f^{m}\right)\right)^{2}$. But for $a \notin V_{m}, \rho_{a}\left(f-f^{m}\right)=\rho_{a}(f)$ and for $a \in V_{m}, \rho_{a}\left(f-f^{m}\right) \leqq 2 \sup \left\{|f(t)-f(u)|, t=u\right.$ in $\left.V_{m}\right\} \leqq 2 \sum_{a \notin V_{m}} \rho_{a}(f)$. Since by assumption $m^{v} \sum_{a \notin V m} \rho_{a}(f) \leqq \sum_{a \notin V_{m}} \rho_{a}(f)|a|^{v}$ converges to zero for $m \rightarrow \infty$, we have $\lim _{m} \lim _{n} \operatorname{Var}\left(S_{n}^{*}(f)-S_{n}^{*}\left(f^{m}\right)\right)_{\mu}=0$. So the theorem follows by a standard argument, see e.g. Billingsley [1], Theorem 4.2.

We can weaken the assumption on the decay of $\gamma_{a}$ and $\rho_{a}(f)$ if we assume instead that the real-valued random field $\left(f \circ \theta_{a}\right)_{a \in \mathbb{Z}^{v}}$ satisfies the FKG inequalities: For all $V \in \mathscr{V}$ and all increasing functions $F, G: \mathbb{R}^{V} \rightarrow \mathbb{R}$ is $\operatorname{Cov}\left(F\left(f \circ \theta_{a}, a \in V\right), G\left(f \circ \theta_{a}\right.\right.$, $a \in V))_{\mu} \geqq 0$. Then the following result is an immediate consequence of Corollary 3.4 and the work of Newman [9].

Theorem 4.2. Let $\mu$ be the unique Gibbs state to a translation invariant, continuous and consistent specification $\left(p^{V}\right)_{V \in \mathcal{V}}$ with $\sum_{a} \gamma_{a}<1$, and let $f$ be in $C(\Omega)$ with $\sum_{a} \rho_{a}(f)<\infty$. If $\left(f \circ \theta_{a}\right)_{a \in \mathbb{Z}^{v}}$ satisfies the $\mathrm{FKG}$ inequalities, then $S_{n}^{*}(f)$ converges weakly to a Gaussian law with mean zero and variance $\sum_{a} \operatorname{Cov}\left(f, f \circ \theta_{a}\right)_{\mu}$. 


\section{Second Derivative of the Pressure}

In this whole section we take $L=\mathbb{Z}^{v}$ and we consider specifications which are given as in (1.9) with the help of a translation invariant potential $\varphi=\left(\varphi_{V}\right)_{V \in \mathcal{V}}$ satisfying (1.8). The set of all such potentials is denoted by $\mathscr{P}$. Taking as norm $\|\varphi\|=\sum_{V \ni 0}|V| \sup \left\{\left|\varphi_{V}(s)\right|, s \in \Omega\right\} \mathscr{P}$ turns into a Banach space. The Dobrushin uniqueness region $\mathscr{D}=\left\{\varphi \in \mathscr{P}, \alpha(\varphi)=\sum_{a} \gamma_{a}(\varphi)<1\right\}$ is then a non-empty open subset of $\mathscr{P}$ (see Gross [7], Proposition 2).

The pressure is defined as usual by

$$
P_{\varphi}=\lim _{V \uparrow \mathbb{Z}^{v}}|V|^{-1} \log \int \exp \left(-\sum_{W \subset V} \varphi_{W}(s)\right) \prod_{a \in V} v\left(d s_{a}\right) .
$$

This limit exists if $V \uparrow \mathbb{Z}^{v}$ is suitably defined, see Ruelle [11]. The main result of this section is the following.

Theorem 5.1. The pressure $P$ is twice continuously differentiable on $\mathscr{D}$. Specifically the second derivative

$$
P_{\varphi}^{\prime \prime}\left(\psi^{1}, \psi^{2}\right)=\left.\frac{\partial^{2} P\left(\varphi+u \psi^{1}+v \psi^{2}\right)}{\partial u \partial v}\right|_{u=v=0}
$$

exists for $\varphi \in \mathscr{D}, \psi^{1}$ and $\psi^{2} \in \mathscr{P}$, and it is equal to

$$
\sum_{a} \operatorname{Cov}\left(f_{\psi^{1}}, f_{\psi^{2}} \circ \theta_{a}\right)_{\mu}
$$

where $f_{\psi^{\prime}}(t)=-\sum_{V \ni 0}|V|^{-1} \psi_{V}^{i}(t)(i=1,2)$ and $\mu$ is the unique Gibbs state in $\mathscr{G}(p(\varphi))$.

Remark 5.2. The existence of the second derivative was already proved by Gross [7], but the identification of the limit as the above covariance series in new. This series converges absolutely by Corollary 3.4 because $\alpha=\gamma$ and $\sum_{a} \rho_{a}\left(f_{\psi}\right)<\infty$, see Gross [7], formula (4.24).

The proof of Theorem 5.1 is based on the following result.

Proposition 5.3. For $\varphi \in \mathscr{D}$ let $\mu_{\varphi}$ be the unique Gibbs state to the potential $\varphi$ and let $g$ be in $C(\Omega)$ with $\sum_{a} \rho_{a}(g)<\infty$. The map $\varphi \rightarrow \mu_{\varphi}(g)$ is then once continuously differentiable on $\mathscr{D}$. Specifically the derivative $\left.\frac{\partial}{\partial u} \mu_{\varphi+u \psi}(g)\right|_{u=0}$ exists for $\varphi \in \mathscr{D}, \psi \in \mathscr{P}$, and it is equal to $\sum_{a} \operatorname{Cov}\left(g, f_{\psi} \circ \theta_{a}\right)_{\mu_{\varphi}}$ where $f_{\psi}$ is as in Theorem 5.1.

Proof. For $\varepsilon>0$ we put $\gamma_{a}=\sup \left\{\gamma_{a}(\varphi+u \psi),|u|<\varepsilon\right\}$, and we choose $\varepsilon$ so small that $\sum_{a} \gamma_{a}<1$. This is always possible, see the proof of Proposition 2 in Gross [7]. We fix $s \in \Omega$ and use for the measure $\pi_{\varphi+u \psi}^{V}(\cdot \mid s)$ the shorter notation $\pi_{u}^{V}$. Similarly we write $\mu_{u}$ instead of $\mu_{\varphi+u \psi}$. For $|u|<\varepsilon$ we have by Corollary 2.4 that $\mu_{u}(g)=\lim _{V \uparrow Z^{v}} \pi_{u}^{V}(g)$. By a simple calculation we get $\frac{\partial}{\partial u} \pi_{u}^{V}(g)=\operatorname{Cov}(g,-$ 
$\sum_{W \cap V \neq \phi} \psi_{W}\left[\pi_{u}^{V}\right)$. Therefore it is sufficient to show that this covariance converges to $\sum_{a} \operatorname{Cov}\left(g, f_{\psi} \circ \theta_{a} \mid \mu_{u}\right)$ uniformly for $|u|<\varepsilon$.

In a first step we replace $-\sum_{W \cap V \neq \phi} \psi_{W}$ by $\sum_{a \in V} f_{\psi}^{\circ} \theta_{a}$ which is equal to - $\sum_{W \cap V \neq \phi} \psi_{W}|W \cap V| /|W|$. Because $p_{u}^{V}(\cdot \mid s)$ is a Gibbs state on $\Omega_{V}=X^{V}$ to the specification $\left(p_{u}^{W}(\cdot \mid \cdot s)\right)_{W \subset v}$, we get from Theorem 3.2 that the error in this step is bounded by

$$
\begin{aligned}
& \sum_{a, b \in V}\left(\sum_{c \in V} \chi_{c a}^{V} \chi_{c b}^{V}\right) \rho_{a}(g) \rho_{b}\left(\sum_{W \cap V \neq \phi}(1-|W \cap V| /|W|) \psi_{W}\right) \\
& \leqq 2 \sum_{a, b \in V}\left(\sum_{c \in V} \chi_{c a}^{V} \chi_{c b}^{V}\right) \rho_{a}(g) \sum_{\substack{W \cap V c \neq \phi \\
W \ni b}} \sup \left\{\left|\psi_{W}(t)\right|, t \in \Omega\right\} .
\end{aligned}
$$

Now the sum over $W$ in the last expression converges to zero for fixed $b$ and $V \uparrow \mathbb{Z}^{\nu}$. Moreover it is surely bounded by $\|\psi\|$ and we can use dominated convergence as in previous proofs in order to see that the error in this first step converges to zero for $V \uparrow \mathbb{Z}^{v}$ uniformly for $|u|<\varepsilon$.

In a second step we replace the expectations with respect to $\pi_{u}^{V}$ by expectations with respect to $\mu_{u}$. For any $V_{0} \subset V$ we split the error into four terms, namely

$$
\begin{aligned}
& \mu_{u}\left(g \cdot \sum_{a \in V_{0}} f_{\psi} \circ \theta_{a}\right)-\pi_{u}^{V}\left(g \cdot \sum_{a \in V_{0}} f_{\psi} \circ \theta_{a}\right), \\
& \mu_{u}(g) \mu_{u}\left(\sum_{a \in V_{0}} f_{\psi} \circ \theta_{a}\right)-\pi_{u}^{V}(g) \pi_{u}^{V}\left(\sum_{a \in V_{0}} f_{\psi} \circ \theta_{a}\right), \\
& \sum_{a \notin V_{0}} \operatorname{Cov}\left(g, f_{\psi} \circ \theta_{a} \mid \mu_{u}\right)
\end{aligned}
$$

and

$$
\sum_{a \in V \backslash V_{0}} \operatorname{Cov}\left(g, f_{\psi}^{\circ} \theta_{a} \mid \pi_{u}^{V}\right)
$$

Using Corollary 2.4, respectively formula (2.4), and similar arguments as before, the terms (5.2) and (5.3) can be shown to be arbitrarily small for fixed $V_{0}$ and $V$ big enough (uniformly for $|u|<\varepsilon$ ). For the terms (5.4) and (5.5) we use Theorem 3.2: they are arbitrarily small if $V_{0}$ is big enough (again uniformly for $|u|<\varepsilon$, the term (5.5) also uniformly in $V \supset V_{0}$ ). So the proof is completed by choosing first a suitable $V_{0}$ and then a, suitable $V$.

Proof of Theorem 5.1. This is now straightforward, see also Gross [7], p. 70. If there is only one Gibbs state $\mu_{\varphi}$, then there is only one tangent functional to the pressure $P$ at $\varphi$ (see e.g. Preston [10], Theorem 8.3), and we have $\left.\frac{\partial}{\partial u} P_{\varphi+u \psi^{1}}\right|_{u=0}=$ $\mu_{\varphi}\left(f_{\psi^{1}}\right)$. So it is sufficient to apply Proposition 5.2 with $g=f_{\psi^{1}}$.

\section{Proofs of the Main Theorems}

We first give a proof of Theorem 2.1 though it will be essentially the same as in 
Dobrushin [4], but we will use an analogous argument for Theorem 3.2 which can be understood more easily in the simpler case of Theorem 2.1. We call $\left(\alpha_{a}\right)_{a \in L}$ an estimate for $\mu_{1}$ and $\mu_{2}, \mu_{i} \in \mathscr{G}\left(p_{i}\right)$, if

$$
\left|\mu_{1}(f)-\mu_{2}(f)\right| \leqq \sum_{a \in L} \alpha_{a} \rho_{a}(f) \quad(f \in C(\Omega)) .
$$

For any $\left(\alpha_{a}\right)_{a \in L}$ and $b \in L$ we define $\left(\tilde{\alpha}_{a}(b)\right)_{a \in L}$ by

$$
\tilde{\alpha}_{a}(b)=\left\{\begin{array}{l}
\alpha_{a} \quad \text { if } a \neq b \\
\beta_{b}+\sum_{c \neq b} \alpha_{c} \gamma_{c b}
\end{array} \quad \text { if } a=b .\right.
$$

The clue for the proof is the following lemma.

Lemma 6.1 If $\left(\alpha_{a}\right)_{a \in L}$ is an estimate for $\mu_{1}$ and $\mu_{2}$, then for any $b \in L\left(\tilde{\alpha}_{a}(b)\right)_{a \in L}$ is also an estimate for $\mu_{1}$ and $\mu_{2}$.

Proof. Using formula (3.5) of Gross [6] we find

$$
\begin{aligned}
\left|\mu_{1}(f)-\mu_{2}(f)\right| \leqq & \left|\mu_{1}\left(\pi_{1}^{b} f\right)-\mu_{1}\left(\pi_{2}^{b} f\right)\right|+\left|\mu_{1}\left(\pi_{2}^{b} f\right)-\mu_{2}\left(\pi_{2}^{b} f\right)\right| \\
\leqq & \beta_{b} \rho_{b}(f)+\sum_{a \in L} \alpha_{a} \rho_{a}\left(\pi_{2}^{b} f\right) \leqq \beta_{b} \rho_{b}(f)+\sum_{a \neq b} \alpha_{a} \rho_{a}(f) \\
& +\sum_{a \neq b} \alpha_{a} \gamma_{a b} \rho_{b}(f) . \quad \square
\end{aligned}
$$

So starting with an arbitrary estimate, e.g. $\alpha_{a} \equiv 1$, one can apply (6.2) repeatedly for different $b \in L$ and hopefully one will reach $\left(\sum_{c} \beta_{c} \chi_{c a}\right)_{a \in L}$ which is not changed by (6.2). However we do not show such a convergence, our proof is indirect: if the best possible estimate is bigger than $\left(\sum_{c} \beta_{c} \chi_{c a}\right)_{a \in L}$, we can always make it smaller by (6.2) which gives a contradiction.

Proof of Theorem 1.2. We fix $V \in \mathscr{V}$ and consider

$$
\alpha_{a}^{S}= \begin{cases}1 & \text { if } a \notin V, \\ S \sum_{c \in V} \beta_{c} \chi_{c a}^{V}+S \sum_{b \notin V, c \in V} \gamma_{b c} \chi_{c a}^{V} \quad \text { if } a \in V .\end{cases}
$$

By the definition of $\chi_{a b}^{V}$ we have

$$
\sum_{a \in V} \chi_{c a}^{V} \gamma_{a b}=\chi_{c b}^{V}-\delta_{c b} \quad(c \in V, b \in V)
$$

which implies for $b \in V$

$$
\begin{aligned}
\sum_{a \in L} \alpha_{a}^{S} \gamma_{a b} & =\sum_{a \notin V} \gamma_{a b}+S \sum_{c \in V} \beta_{c} \chi_{c b}^{V}-S \beta_{b}+S \sum_{d \notin V, c \in V} \gamma_{d c} \chi_{c b}^{V} \\
-S \sum_{d \notin V} \gamma_{d b} & =\alpha_{b}^{S}-S \beta_{b}-(S-1) \sum_{d \notin V} \gamma_{d b} .
\end{aligned}
$$

Let us assume that $\beta_{a}>0$ for all $a \in L$. This is no essential restriction since we can always consider first $\widetilde{\beta}_{a}=\beta_{a}+\varepsilon$ and then let $\varepsilon$ tend to zero. Under this assumption 
$\left(\alpha_{a}^{S}\right)_{a \in L}$ is an estimate for $\mu_{1}$ and $\mu_{2}$ if $S \geqq \max \left(\beta_{a}^{-1}, a \in V\right)$. We put $\bar{S}=\inf \{S$, $\left(\alpha_{a}^{S}\right)_{a \in L}$ is an estimate for $\mu_{1}$ and $\left.\mu_{2}\right\}<\infty$. We suppose that $\bar{S}>1$ and show that this leads to a contradiction.

To any estimate $\left(\alpha_{a}\right)_{a \in L}$ for $\mu_{1}$ and $\mu_{2}$ satisfying $\alpha_{a} \leqq \alpha_{a}^{\bar{S}(1+\delta)}(a \in L)$ for some $\delta>0$ there is by the definition of $\bar{S}$ a point $b \in V$ such that $\alpha_{b}>\alpha_{b}^{\bar{S}(1-\delta)}$. To any such estimate we consider the estimate $\left(\tilde{\alpha}_{a}(b)\right)_{a \in L}$ defined by (6.2). Then we get from

$$
\tilde{\alpha}_{b}(b) \leqq \beta_{b}+\sum_{a \in L} \alpha_{a}^{\bar{S}(1+\delta)} \gamma_{a b} \leqq \alpha_{b}^{\bar{S}(1-\delta)}+2 \bar{S} \delta \alpha_{b}^{1}-(\bar{S}(1+\delta)-1)\left(\beta_{b}+\sum_{d \notin V} \gamma_{d b}\right) \leqq
$$
$\alpha_{b}^{\bar{S}(1-\delta)}$ if $\delta$ is small enough because $\bar{S}>1$. So by repeated application of (6.2) we can find an estimate satisfying $\alpha_{a} \leqq \alpha_{a}^{\bar{S}(1-\delta)}$ for all $a \in V$ and therefore also for all $a \in L$ which contradicts the definition of $\bar{S}$. Hence $\bar{S} \leqq 1$.

Finally we want to expand $V$. This can be done easily because $\chi_{a b}^{V}$ increases to $\chi_{a b}$ for $V \uparrow L$ and for fixed $a \in L \sum_{b \notin V, c \in V} \gamma_{b c} \chi_{c a}^{V}$ tends to zero, see the argument in the proof of Proposition 1.4.

Turning now to the proof of Theorem 3.2 we call $\left(\alpha_{a b}\right)_{a, b \in L}$ with $\alpha_{a b}=\alpha_{b a}$ a covariance estimate for $\mu \in \mathscr{G}(p)$ if

$$
\left|\operatorname{Cov}(f, g)_{\mu}\right| \leqq \sum_{a, b \in L} \alpha_{a b} \rho_{a}(f) \rho_{b}(g) \quad(f, g \in C(\Omega))
$$

$\alpha_{a b} \equiv 1$ is always a covariance estimate for any $\mu$ because $\inf f(s) \leqq \mu(f) \leqq \sup f(s)$. The analogue of $(6.2)$ is

$$
\tilde{\alpha}_{a b}(c)=\left\{\begin{array}{l}
\alpha_{a b} \quad \text { if } a \neq c \text { and } b \neq c \\
\sum_{a^{\prime}} \alpha_{a a^{\prime}} \gamma_{a^{\prime} c} \quad \text { if } a \neq c \text { and } b=c, \\
\sum_{a^{\prime}} \alpha_{b a^{\prime}} \gamma_{a^{\prime} c} \quad \text { if } a=c \text { and } b \neq c, \\
\sum_{a^{\prime}, b^{\prime}} \alpha_{a^{\prime} b^{\prime}} \gamma_{a^{\prime} c} \gamma_{b^{\prime} c}+1 \quad \text { if } a=b=c .
\end{array}\right.
$$

and the following lemma corresponds to Lemma 6.1 :

Lemma 6.2. If $\left(\alpha_{a b}\right)_{a, b \in L}$ is a covariance estimate for $\mu$, then for any $c \in L\left(\tilde{\alpha}_{a b}(c)\right)_{a, b \in L}$ is also a covariance estimate for $\mu$.

Proof. $\left|\operatorname{Cov}(f, g)_{\mu}\right| \leqq\left|\mu\left(\pi^{c}(f g)\right)-\mu\left(\pi^{c} f \pi^{c} g\right)\right|+\left|\mu\left(\pi^{c} f \pi^{c} g\right)-\mu\left(\pi^{c} f\right) \mu\left(\pi^{c} g\right)\right| \leqq \rho_{c}(f) \rho_{c}(g)$

$+\sum_{a, b} \alpha_{a b} \rho_{a}\left(\pi^{c} f\right) \rho_{b}\left(\pi^{c} g\right) \leqq \rho_{c}(f) \rho_{c}(g)+\sum_{a \neq c, b \neq c} \alpha_{a b} \rho_{a}(f) \rho_{b}(g)+\sum_{a \neq c, b} \alpha_{a b} \gamma_{b c} \rho_{a}(f) \rho_{c}(g)$,

$+\sum_{b \neq c, a} \alpha_{a b} \gamma_{a c} \rho_{c}(f) \rho_{b}(g)+\sum_{a, b} \alpha_{a b} \gamma_{b c} \gamma_{a c} \rho_{c}(f) \rho_{c}(g)$, and we note that $\alpha_{a b}=\alpha_{b a}$.

The proof of Theorem 3.2 follows now the same lines as the previous proof. The only difference is that $\gamma^{*}\left(\sum_{c} \chi_{c a} \chi_{c b}\right)_{a, b \in L}$ with $\gamma^{*}$ defined by (3.3) does not remain unchanged by (6.7), but it is at least not increased by (6.7) which is sufficient for us (see also Remark 6.3 below). 
Proof of Theorem 3.2. We fix $V$, put $M=1 / \gamma^{*}$ and consider

$$
\alpha_{a b}^{S}=\left\{\begin{array}{l}
1 \quad \text { if } a \notin V \text { or } b \notin V \\
S \sum_{c \in V} \chi_{c a}^{V} \chi_{c b}^{V}+M \cdot S \sum_{c \notin V, d \in V} \gamma_{c d}\left(\chi_{d a}^{V}+\chi_{d b}^{V}\right) \quad \text { if } a \text { and } b \in V .
\end{array}\right.
$$

Then using (6.4) we find for $a \in V, b \in V$

$$
\begin{aligned}
\sum_{c} \alpha_{a c}^{S} \gamma_{c b}= & \sum_{c \notin V} \gamma_{c b}+S \sum_{c^{\prime} \in V} \chi_{c^{\prime} a}^{V} \chi_{c^{\prime} b}^{V}-S \cdot \chi_{b a}^{V}+M \cdot S \sum_{c^{\prime} \notin V, d \in V} \gamma_{c^{\prime} d} \chi_{d a}^{V} \cdot \sum_{c \in V} \gamma_{c b} \\
& +M \cdot S \sum_{c^{\prime} \in V, d \in V} \gamma_{c^{\prime} d} \chi_{d b}^{V}-M \cdot S \sum_{c^{\prime} \notin V} \gamma_{c^{\prime} b} \leqq \alpha_{a b}^{S}-S \cdot \chi_{b a}^{V} \\
& -(M \cdot S-1) \sum_{c \notin V} \gamma_{c b},
\end{aligned}
$$

since $\sum_{c \in V} \gamma_{c b}<1$. From (6.9) it follows that for $b \in V$

$$
\begin{aligned}
\sum_{a, c} \alpha_{a c}^{S} \gamma_{a b} \gamma_{c b} \leqq & \sum_{a \notin V} \gamma_{a b} \sum_{c} \gamma_{c b}+\sum_{a \in V} \alpha_{a b}^{S} \gamma_{a b}-S \sum_{a \in V} \chi_{b a}^{V} \gamma_{a b}-(M \cdot S-1) \\
& \cdot \sum_{c \notin V} \gamma_{c b} \sum_{a \in V} \gamma_{a b} \leqq \sum_{a \notin V} \gamma_{a b}+\alpha_{b b}^{S}-S \chi_{b b}^{V}-M \cdot S \sum_{c \notin V} \gamma_{c b} \\
& -S \chi_{b b}^{V}+S-(M \cdot S-1) \sum_{c \notin V} \gamma_{c b}=\alpha_{b b}^{S}-1-\left(S\left(2 \chi_{b b}^{V}-1\right)-1\right) \\
& -2(M \cdot S-1) \sum_{c \notin V} \gamma_{c b}
\end{aligned}
$$

(we have used $\alpha_{a b}^{S}=\alpha_{b a}^{S}$ and $\sum_{c} \gamma_{c b}<1$ ). The important point is that for $S>\gamma^{*}$ the final bound in (6.9) and ${ }^{c}(6.10)$ is less than $\alpha_{a b}^{S}$ respectively $\alpha_{b b}^{S}-1$ if we assume that $\chi_{a b}^{v}>0$ for all $a \in V, b \in V$. If this assumption does not hold we can take first $\tilde{\gamma}_{a b}=\gamma_{a b}+\varepsilon \beta_{a b}$ for a suitable $\left(\beta_{a b}\right)$ and then let $\varepsilon$ go to zero.

We put $\bar{S}=\inf \left\{S,\left(\alpha_{a b}^{S}\right)_{a, b \in L}\right.$ is a covariance estimate for $\left.\mu\right\}<\infty$. Suppose that $\bar{S}>\gamma^{*}$ and let $\left(\alpha_{a b}\right)_{a, b \in L}$ be any covariance estimate for $\mu$ satisfying $\alpha_{a b} \leqq \alpha_{a b} \bar{S}^{(1+\delta)}$ $(a, b \in L)$ for some $\delta>0$. By definition of $\bar{S}$ there is then a pair $a_{0} \in V, b_{0} \in V$ such that $\alpha_{a_{0} b_{0}}>\alpha_{a_{0} b_{0}}^{\bar{S}(1-\delta)}$. To such an estimate we consider the estimate $\left(\tilde{\alpha}_{a b}\left(a_{0}\right)\right)$ defined by (6.7). From (6.9), (6.10) and $\sum_{c} \gamma_{c b}<1$ we get then $\tilde{\alpha}_{a_{0} b}\left(a_{0}\right)=\tilde{\alpha}_{b a_{0}}\left(a_{0}\right) \leqq$ $\alpha_{a_{0} b}^{\bar{S}(1-\delta)}(b \in L)$ for $\delta$ small enough. So by repeated application of (6.7) we come to a contradiction like in the previous proof.

Finally for letting $V$ tend to $L$ we argue as before.

Remark 6.3. It is a little disturbing that the estimate $\left(\gamma^{*} \sum_{c} \chi_{c a} \chi_{c b}\right)_{a, b \in L}$ is not optimal because it can still be made smaller by (6.7). In the case $\gamma_{a b}=\gamma_{b a}(a, b \in L)$, e.g. if we have pair interactions, $\left(\chi_{a b}\right)_{a, b \in L}$ is unchanged by (6.7), and the same proof as before shows that then $\left(\chi_{a b}\right)_{a, b \in L}$ is also a covariance estimate for $\mu$. It can be proved that always $\chi_{a b} \leqq \gamma^{*} \sum_{c} \chi_{c a} \chi_{c b}(a, b \in L)$, however this new estimate gives in 
the Corollaries 3.3 and 3.4 only the smaller constant $(1-\alpha)^{-1}$ instead of $\gamma^{*}(1-\alpha)^{-2}$, but no substantial improvement.

\section{Generalization to the Non-Compact case}

Let $(X, \mathscr{B})$ be a measurable space with a metric $r(\cdot, \cdot)$ which is a measurable function on $(X, \mathscr{B}) \times(X, \mathscr{B})$. For two probabilities $q_{1}$ and $q_{2}$ on $(X, \mathscr{B})$ the Vasershtein distance is defined by

$$
R\left(q_{1}, q_{2}\right)=\inf \int r\left(s_{1}, s_{2}\right) Q\left(d s_{1}, d s_{2}\right),
$$

where the infimum is taken over all probabilities $Q$ on $(X, \mathscr{B}) \times(X, \mathscr{B})$ with projections $q_{1}$ and $q_{2}$. For the metric $r\left(s_{1}, s_{2}\right)=0$ if $s_{1}=s_{2}, r\left(s_{1}, s_{2}\right)=1$ otherwise, we have $R\left(q_{1}, q_{2}\right)=\frac{1}{2}\left\|q_{1}-q_{2}\right\|_{\text {Var }}$. For two specifications $\left(p_{i}^{V}\right)_{V \in \mathscr{V}}, i=1,2$, on $\Omega=X^{L}$ we define

$$
\begin{aligned}
& \gamma_{a b}=\sup \left\{R\left(p_{i}^{b}(\cdot \mid s), p_{i}^{b}(\cdot \mid t)\right) / r\left(s_{a}, t_{a}\right), s=t \text { expect at } a, i=1,2\right\} \\
& \beta_{a}=\sup \left\{R\left(p_{1}^{a}(\cdot \mid s), p_{2}^{a}(\cdot \mid s)\right), s \in \Omega\right\},
\end{aligned}
$$

The role of $C(\Omega)$ is taken over by the "Lipschitz continuous" functions: For $f: \Omega \rightarrow \mathbb{R}$ we put

$$
\rho_{a}(f)=\sup \left\{|f(s)-f(t)| / r\left(s_{a}, t_{a}\right), s=t \text { expect at } a\right\}
$$

and let $L C(\Omega)$ be the set of functions for which $\rho_{a}(f)<\infty$

$$
(a \in L) \text { and }|f(s)-f(t)| \leqq \sum_{a} \rho_{a}(f) r\left(s_{a}, t_{a}\right) \quad(s \in \Omega, t \in \Omega) .
$$

Like Theorem 2.1 the next result is essentially in Dobrushin [4].

Theorem 7.1. Suppose $\left(p_{i}^{V}\right)_{V \in \mathcal{F}}, i=1,2$, are two specifications such that $\sum_{a} \gamma_{a b} \leqq$ $\alpha<1$ and $\pi_{i}^{V}(L C(\Omega)) \subset L C(\Omega)$. Let $\mu_{i}$ be in $\mathscr{G}\left(p_{i}\right)$ such that for some $u \in \Omega \int r\left(s_{a}, u_{a}\right)$ $\mu_{i}(d s) \leqq C<\infty(i=1,2)$. Then we have for all $f \in L C(\Omega)$

$$
\left|\mu_{1}(f)-\mu_{2}(f)\right| \leqq \sum_{a, b} \beta_{b} \chi_{b a} \rho_{a}(f)
$$

Proof. By the definition of $L C(\Omega)$ we have for $f \in L C(\Omega)$ :

$$
\begin{aligned}
\left|\mu_{1}(f)-\mu_{2}(f)\right| & \leqq \iint|f(s)-f(t)| \mu_{1}(d s) \mu_{2}(d t) \\
& \leqq \sum_{a} \rho_{a}(f)\left(\int r\left(s_{a}, u_{a}\right) \mu_{1}(d s)+\int r\left(u_{a}, t_{a}\right) \mu_{2}(d t)\right) \\
& \leqq 2 C \sum_{a} \rho_{a}(f),
\end{aligned}
$$

so there exists a uniformly bounded estimate for $\mu_{1}$ and $\mu_{2}$. The rest of the proof, in particular Lemma 6.1, is the same. For details see Dobrushin [4].

For the generalization of Theorem 3.2 we need one more definition. Let

$$
\sigma_{a}^{2}=\sup \left\{\inf \left\{\int r(u, m(s))^{2} p^{a}(d u \mid s), m: X^{L \backslash a} \rightarrow X\right\}, \quad s \in X^{L \backslash a}\right\} .
$$


Theorem 7.2. Suppose $\left(p^{V}\right)_{V \in \mathscr{V}}$ is a specification such that $\sum_{a} \gamma_{a b} \leqq \alpha<1, \sigma_{a}^{2} \leqq$ $\sigma^{2}<\infty$ and $\pi^{V}(L C(\Omega)) \subset L C(\Omega)$. Let $\mu$ be in $\mathscr{G}(p)$ such that for some $u \in \Omega \int r\left(s_{a}, u_{a}\right)^{2}$ $\mu(d s) \leqq C<\infty$. Then we have for all $f$ and $g$ in $L C(\Omega)$

$$
\left|\operatorname{Cov}(f, g)_{\mu}\right| \leqq 4 \sigma^{2} \gamma^{*} \sum_{a, b, c} \chi_{c a} \chi_{c b} \rho_{a}(f) \rho_{b}(g)
$$

Proof. First we observe that

$$
\begin{aligned}
\left|\operatorname{Cov}(f, g)_{\mu}\right| \leqq & \iiint|f(s)-f(t)||g(s)-g(v)| \mu(d s) \mu(d t) \mu(d v) \\
\leqq & \sum_{a, b} \rho_{a}(f) \rho_{b}(g) \iiint \int\left(r\left(s_{a}, u_{a}\right)+r\left(u_{a}, t_{a}\right)\right)\left(r\left(s_{b}, u_{b}\right)+r\left(u_{b}, v_{b}\right)\right) \\
& \cdot \mu(d s) \mu(d t) \mu(d v) \leqq 4 C \sum_{a, b} \rho_{a}(f) \rho_{b}(g),
\end{aligned}
$$

so there exists a uniformly bounded covariance estimate for $\mu$. Furthermore

$$
\begin{aligned}
\left|\mu\left(\pi^{c}(f g)\right)-\mu\left(\pi^{c} f \pi^{c} g\right)\right| & \leqq \rho_{c}(f) \rho_{c}(g) \sup _{s} \iiint r(u, t) r(u, v) p^{c}(d u \mid s) p^{c}(d t \mid s) p^{c}(d v \mid s) \\
& \leqq 4 \sigma_{c}^{2} \rho_{c}(f) \rho_{c}(g),
\end{aligned}
$$

so Lemma 6.2 will be correct if we define $\tilde{\alpha}_{c c}(c)$ in (6.7) by $\sum_{a^{\prime}, b^{\prime}} \alpha_{a^{\prime} b^{\prime}} \gamma_{a^{\prime} c} \gamma_{b^{\prime} c}+4 \sigma^{2}$.

The rest of the proof is the same.

Acknowledgement. I wish to express my gratitude to $\mathrm{H}$. Föllmer whose lectures introduced me to the subject. Thanks are also due to L. Gross who pointed out to me the open problem on the second derivative of the pressure.

\section{References}

1. Billingsley, P.: Convergence of probability measures. New York, London, Sidney, Toronto: Wiley 1968

2. Bolthausen, E.: A note on the central limit theorem for stationary random fields. Preprint Berlin (1980)

3. Dobrushin, R. L.: The description of a random field by means of conditional probabilities and conditions of its regularity. Theor. Probab. Appl. 13, 197-224 (1968)

4. Dobrushin, R. L.: Prescribing a system of random variables by conditional distributions. Theor. Probab. Appl. 15, 458-486 (1970)

5. Dobrushin, R. L., Tirozzi, B.: The central limit theorem and the problem of equivalence of ensembles. Commun. Math. Phys. 54, 173-192 (1977)

6. Gross, L.: Decay of correlations in classical lattice models at high temperature. Commun. Math. Phys. 68, 9-27 (1979)

7. Gross, L.: Absence of second-order phase transitions in the Dobrushin uniqueness region. J. Stat. Phys. 25, 57-72 (1981)

8. Nahapetian, B. S.: The central limit theorem for random fields with mixing property. In: Multicomponent random systems, Dobrushin, R. L., Sinai, Ya. G. (eds.). New York, Basel: Dekker 1980

9. Newman, C. M.: Normal fluctuations and the FKG inequalities. Commun. Math. Phys. 74, 119-128 (1980)

10. Preston, C.: Random fields. In: Lecture notes in Mathematics, Vol. 534. Berlin, Heidelberg, New York: Springer 1976

11. Ruelle, D.: Statistical mechanics. New York: Benjamin 1969 
12. Simon, B.: A remark on Dobrushin's uniqueness theorem. Commun. Math. Phys. 68, 183-185 (1979)

13. Vasershtein, L. N.: Markov processes over denumerable products of spaces, describing large systems of automata. Probl. Inf. Transm. 5, 64-72 (1969)

Communicated by E. Lieb

Received September 1, 1981 